\title{
A Case Report of a Pheochromocytoma Presenting With NeUrological Manifestations
}

\author{
Nazia Shamsuddin, MD, Whitney Jackson, $M D$
}

\section{Case Report}

A 51 year-old Caucasian female with a past medical history of hypertension, coronary artery disease and cerebrovascular accident presented to the emergency room with acute onset dysarthria and right-sided hemiparesis. The patient reported that she noticed these symptoms when she woke that morning, but had resolved by the time she reached the emergency room, an hour later. She denied experiencing palpitations, headache, chest pain or shortness of breath.

On review of systems, it was revealed she had intermittent nausea and vomiting for a year with increasing frequency over the past two days. She denied blood or bile in the emesis. She also admitted to a 50-pound weight loss over the past year. Both esophagogastroduodenoscopy and colonoscopy were normal within the past year.

Her medical history was significant for coronary artery disease complicated by two myocardial infarctions the previous year. She had also suffered from two past cerebrovascular accidents - the second of which was complicated by hemorrhage and seizure. Despite several anti-hypertensive medications, the patient's blood pressure remained uncontrolled.

On admission, the patient was afebrile, had an elevated blood pressure of $167 / 108$, pulse of 95 beats/minute, respiratory rate of 18 breathes/minute, and pulse oximetry of $97 \%$ on room air. Generally, the patient appeared comfortable. Cardiovascular exam was benign and pulmonary exam revealed decreased breath sounds bilaterally. Abdominal exam was also benign with no masses appreciated. Neurological exam was significant for slight dysarthria, right homonymous hemianopsia, and right-sided facial droop. Upper and lower extremity strength testing was significant for weakness at $4 / 5$ strength on the left side. Comparatively, the patient's strength was $5 / 5$ in the upper and lower extremities on the right side. Additionally, she was hyper-reflexive on the right side compared to the left. Sensation was intact throughout. These neurological deficits were consistent with her baseline deficits from past strokes.

CT of the head was significant for an old left middle cerebral artery stroke, but there was no evidence of an acute process. A CT of the abdomen was performed in the emergency room, which revealed an incidental finding of a $6.7 \times 5.4 \mathrm{~cm}$ right sided adrenal mass (Figure 1).

The laboratory work-up revealed elevated catecholamines: plasma norepinephrine of 41.6, plasma metanephrine of 27.2, fractionated urine norepinephrine of 9390 and fractionated urine metanephrine of 8231 .

Diagnoses of transient ischemic attack and pheochromocytoma were made. Her blood pressure medications were changed to the alpha-blocker, phenoxybenzamine. A beta-blocker was later added for additional blood pressure control. The mass was surgically removed four weeks later.

\section{Discussion}

A pheochromocytoma is a tumor that results in excess secretion of the catecholamines epinephrine and norepinephrine. It arises from chromaffin cells of the medulla of the adrenal gland, but it can also be located in extra-adrenal, retroperitoneal, pelvic or thoracic sites. Diagnosis relies on measurement of plasma levels of free metanephrines. This test has a high sensitivity (99\%) and specificity $(89 \%)^{1}$. Between episodes of catecholamine release, catecholamine levels may be normal, which is why the recommended test measures metabolites, rather than the catecholamines themselves. The sensitivity and specificity of other tests such as urinary catecholamines are high, but still less sensitive and less specific than measuring plasma free metanephrines ${ }^{1}$.

Although pheochromocytomas are rare, autopsy studies suggest a higher prevalence. The National Cancer Registry in Sweden has reported that pheochromocytomas are discovered in two patients per million people each year ${ }^{2}$. Interestingly, in autopsy studies, the prevalence of adrenal masses may be as high as $8 \%^{3}$, and of these, $4.2 \%$ are diagnosed as pheochromocytomas ${ }^{1}$.

Due to its variable clinical presentation, pheochromocytomas have been called "the masquerader". Retrospective studies show that of people with pheochromocytomas at time of autopsy, $61 \%$ had a history of hypertension and $91 \%$ had a history of "typical" symptoms, generally considered to be headaches, palpitations and sweating, but atypical symptoms have also been described. These atypical symptoms include: abdominal pain, nausea, vomiting, and dyspnea. Causes of death in people diagnosed with pheochromocytoma incidentally at time of autopsy include: myocardial infarction, cerebrovascular accident, arrhythmias, shock, renal failure and dissecting aortic aneurysm ${ }^{2}$. The catecholamines released by pheochromocytomas can lead to heart failure, pulmonary edema, arrhythmias, and intracranial hemorrhage. A pheochromocytoma presenting with cerebrovascular injury is rare and the incidence is unknown.

There are two proposed mechanisms for neurological injury resulting from a pheochromocytoma: hypertension and vasospasm. During excess catecholamine release, high blood pressure may overwhelm cerebrovascular autoregulation leading to hypertensive encephalopathy. The second proposed mechanism suggests that catecholamine excess or sympathomimetics cause spasm of the cerebral arteries. These vascular spasms can cause infarction or transient impairment of circulation ${ }^{4,5}$.

Management of cerebrovascular injury involves inhibiting the effects of the released catecholamines, epinephrine and norepinephrine. When these hormones are released, epinephrine 


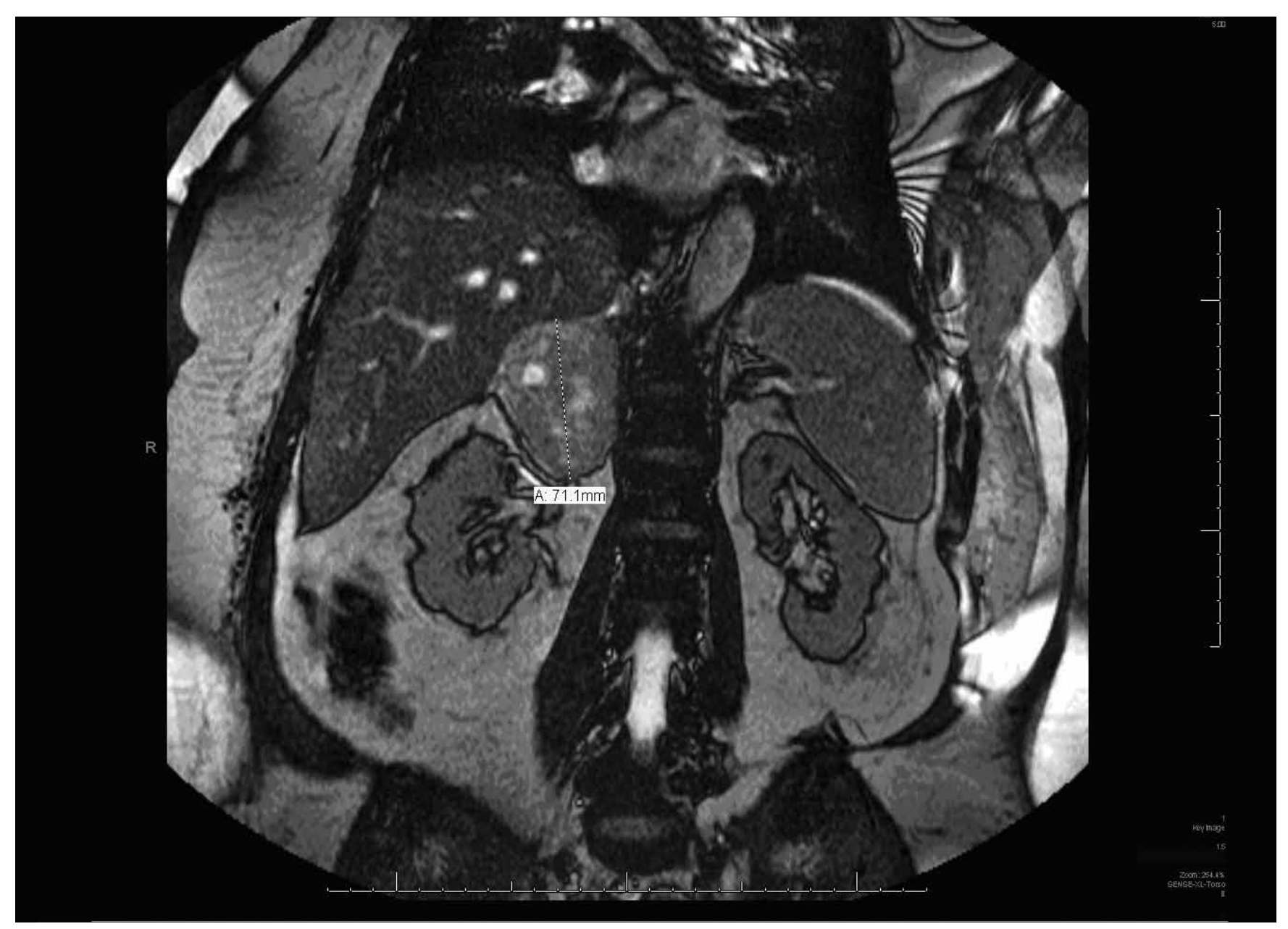

Figure 1. CT scan of the abdomen showing $6.7 \times 5.4 \mathrm{~cm}$ adrenal mass.

acts on alpha and beta adrenergic receptors while norepinephrine acts on the same receptors, except $\beta 2$ adrenergic receptors. The cumulative effect is potent peripheral vasoconstriction by alpha receptor agonism and increased heart rate by $\beta 1$ agonism $^{6}$. Thus, management of hypertension due to a secondary cause like pheochromocytoma is very specific. Pre-operative medical management includes initial alpha antagonism followed by beta antagonism. Of importance, an alpha blocker such as Phenoxybenzamine is recommended approximately 2 days before starting beta-blockade agents ${ }^{7}$. The rationale is that beta-blockade alone would result in blocking of beta receptors that cause peripheral vasodilation, leaving alpha mediated peripheral vasoconstriciton unopposed. Additionally, Metyrosine, a competitive inhibitor of the enzyme needed for catecholamine synthesis, has been proposed for pheochromocytoma management but is rarely clinically utilized at this time $e^{6}$.

Definitive treatment of a pheochromocytoma is surgical removal, which is curative in up to $90 \%$ of cases ${ }^{1}$. Prior to surgery, the patient's clinical status is optimized with blood pressure control and volume repletion to avoid the consequences of the stress response from anesthesia and the surgery itself, which could involve a massive release of catecholamines.

\section{References}

1. Pacak K. Linehan WM. Eisenhofer G. Walther MM. Goldstein DS. Recent advances in genetics, diagnosis, localization, and treatment of pheochromocytoma. Ann Intern Med. 34(4):315-29, 2001 Feb 20.

2. Fitzgerald Paul A, "Chapter 12. Adrenal Medulla \& Paraganglia” (Chapter). Gardner DG, Shoback D: Greenspan's Basic and Clinical Endocrinology, 8e: http://www.accessmedicine.com.proxyl.lib.tju.edu:2048/content. aspx?aID=2630008.

3. Sutton MG, Sheps SG, Lie JT. Prevalence of clinically unsuspected pheochromocytoma. Review of a 50-year autopsy series. Mayo Clin Proc. 56(6):354-60, Jun 1981.

4. Lin PC, Hsu JT, Chung CM, Chang ST. Pheochromocytoma underlying hypertension, stroke, and dilated cardiomyopathy. Tex Heart Inst J. 34(2):244-6, 2007.

5. Razavi M, Bendixen B, Maley JE, Shoaib M, Zargarian M, Razavi B, Adams HP. CNS pseudovasculitis in a patient with pheochromocytoma. Neurology. 52(5):1088-90, Mar 1999.

6. Pacak, K. Preoperative Management of the Pheochromocytoma Patient. Journal of Clinical Endocrinology and Metabolism. Nov 2007: 92(11) 4069 - 4079.

7. Pacak K, Eisenhofer G, Ahlman H, Bornstein SR, Gimenez-Roqueplo AP, Grossman AB, Kimura N, Mannelli M, McNicol AM, Tischler AS. Pheochromocytoma: recommendations for clinical practice from the First International Symposium. Nat Clin Pract Endocrinol Metab. Feb 2007: 3(2) 92-102. 